\title{
Spontaneous Generation of Self-Organized Solitary Wave Structures at Earth's Magnetopause
}

\author{
R. Trines, ${ }^{1}$ R. Bingham, ${ }^{1, *}$ M. W. Dunlop, ${ }^{1}$ A. Vaivads, ${ }^{2}$ J. A. Davies, ${ }^{1}$ J. T. Mendonça, ${ }^{3}$ L. O. Silva, ${ }^{3}$ and P. K. Shukla ${ }^{4}$ \\ ${ }^{1}$ Rutherford Appleton Laboratory, Chilton, Didcot, Oxon, OX11 OQX, United Kingdom \\ ${ }^{2}$ Swedish Institute for Space Physics, Box 537, SE-751 21 Uppsala, Sweden \\ ${ }^{3}$ Instituto Superior Técnico, Av. Rovisco Pais, 1049-001 Lisbon, Portugal \\ ${ }^{4}$ Ruhr-Universität Bochum, 44780 Bochum, Germany \\ (Received 31 May 2007; published 16 November 2007)
}

\begin{abstract}
Spontaneous formation of solitary wave structures has been observed in Earth's magnetopause, and is shown to be caused by the breakup of a zonal flow by the action of drift wave turbulence. Here we show matched observations and modeling of coherent, large-scale solitary electrostatic structures, generated during the interaction of short-scale drift wave turbulence and zonal flows at the Earth's magnetopause. The observations were made by the Cluster spacecraft and the numerical modeling was performed using the wave-kinetic approach to drift wave-zonal flow interactions. Good agreement between observations and simulations has been found, thus explaining the emergence of the observed solitary structures as well as confirming earlier theoretical predictions of their existence.
\end{abstract}

Soliton formation is a common phenomenon in the evolution of nonlinear waves and turbulence. Well-known examples are soliton formation in nonlinear optics and solitary waves in fluids and plasmas [1,2]. In the study of magnetically confined plasmas such as tokamaks, the interaction of zonal flows and drift mode turbulence, described by the Charney-Hasegawa-Mima equations [36], can cause the generation of solitary wave structures [7]. Similar phenomena occur in the coupling between Rossby waves and zonal flows in planetary atmospheres [8].

Recent observations by the Cluster satellites at the magnetopause [9-11], which is the boundary separating the shocked solar wind and Earth's magnetosphere, demonstrate the existence of electrostatic solitary wave structures moving down the ion density gradient associated with this boundary. These structures coincide with much higher frequency modes around the lower-hybrid drift frequency, and theory suggests that the two are associated with each other [7]. In this Letter we demonstrate that the lowfrequency electrostatic solitary structures originate from the breakup of zonal flows. These zonal flows are driven by the higher frequency drift modes through wave collapse [12] of the drift modes. We have applied, for the first time, the numerical model of the modulational interaction of drift modes and zonal flows to real experimental data. Numerical simulations of the wave collapse instability of drift waves, adapted for the Cluster context, show good agreement with the observations. In both theory and simulations, the drift mode turbulence exhibits strong wave collapse leading to spontaneous formation of solitary wave structures which move rapidly down the magnetopause plasma density gradient, penetrating into the magnetosphere to different depths at different sampling times.

The observations analyzed here were made by the fleet of four ESA Cluster spacecraft [9], which provide unique, multipoint measurements of the Earth's magnetosphere. Measurements were taken during an outbound pass through the magnetopause boundary layer, which contains a current layer that separates the shocked solar wind plasma, the magnetosheath, from plasma trapped by the closed magnetic field of the Earth, the magnetosphere. The event we analyze occurred at the magnetopause boundary layer on 30 March 2002, and has been reported by Keyser et al., [10] and Silin et al. [11], who identified the electric field turbulence within the current layer to consist of lowerhybrid drift waves. The orbit and configuration of the spacecraft are given in Fig. 1, which shows that, during the event, Cluster moves from the tail lobe of the magnetosphere into the magnetosheath. As indicated in the figure, the spacecraft were on an outbound pass through the magnetopause when the event described here occurred. Figure 2 displays an overview of the measurements taken by Cluster $[9,11]$. Clockwise, the top four panels show the component of the magnetic field parallel to the boundary (which defines the extent and maximum value of the current layer), the background plasma density (which defines the boundary between the magnetosheath and magnetosphere plasmas), and the ac $(20-180 \mathrm{~Hz})$ and dc $(0-20$ $\mathrm{Hz}$ ) components of the boundary normal electric field. The framed region of interest in the electric field graphs is shown magnified in the two panels at the bottom of the figure, clearly showing solitary waves in the dc field, and modulated wave packets in the ac field.

The Cluster observations displayed in Fig. 2 were taken on 30 March, 2002, between 13:11:40 and 13:11:47 UT. The field data have been taken by the EFW (electric field) [13] and FGM (magnetic field) [14] instruments of the Cluster spacecraft, while the plasma density has been derived from the spacecraft potential [15]. Note that the electric field signal for $\mathrm{C} 1$ (black curves in Fig. 2) was unavailable when the observations were made. The zero- 


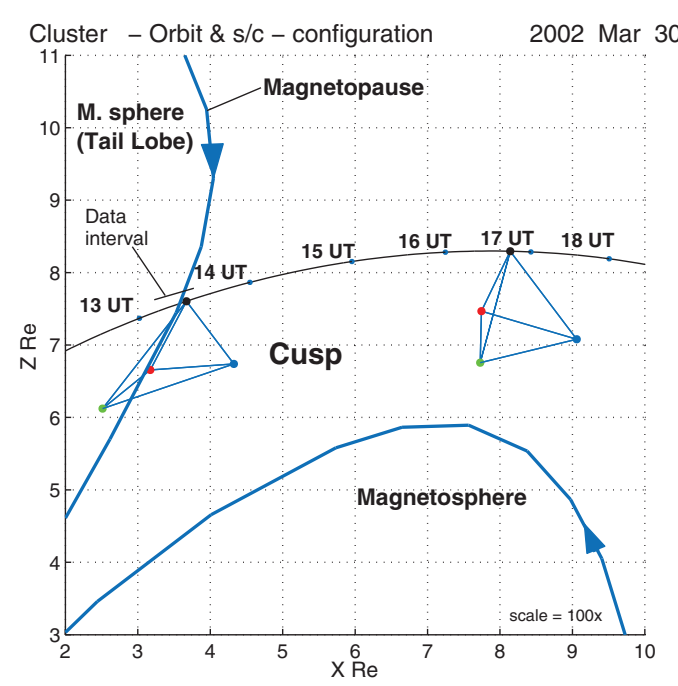

FIG. 1 (color). Configuration of the four cluster spacecraft on an outbound pass through the magnetopause, at the time the observations were made. The figure shows the projection on a plane containing the Earth's axis of rotation. The heavy blue lines denote the magnetic field lines close to the polar cusp. The color coding is black for $\mathrm{C} 1$, red for $\mathrm{C} 2$, green for $\mathrm{C} 3$, and blue for $\mathrm{C} 4$, while the black curve with blue dots denotes the orbit of $\mathrm{C} 1$, which is taken as a reference for the orbit of the entire Cluster system. Distances are measured in Earth radii. The pictured size of the tetrahedron is 100 times the actual size.

crossing of the $B_{L}$-component of the magnetic field took place at 13:11:45 UT for spacecraft $\mathrm{C} 1$, and defines the instant of the spacecraft entering the magnetopause boundary layer from the magnetosphere, as shown in the top left frame of Fig. 2 (with the magnetosphere on the left, magnetopause on the right). In order to line up the boundary layer crossings of all four spacecraft and to highlight the boundary layer traversed by each spacecraft, a time delay has been imposed on signals provided by $\mathrm{C} 2$ (red, $-2.1 \mathrm{~s}$ ), C3 (green, $-3.1 \mathrm{~s}$ ) and C4 (blue, $+0.5 \mathrm{~s}$ ) to match them to $\mathrm{C} 1$ (black). This time delay also takes care of systematic errors in the timing of the individual spacecraft, which otherwise amount roughly to $0.1 \mathrm{~s}$.

In the following, the plasma electron temperature is denoted by $T_{e}$, the ion (proton) mass by $m_{i}$, the ion sound speed by $c_{s}=\sqrt{T_{e} / m_{i}}$, the Earth's geomagnetic field by $B$, the ion gyro-frequency by $\Omega_{c i}=e B / m_{i}$ where $e$ is the magnitude of the electron charge, and the ion sound gyroradius by $\rho_{s}=c_{s} / \Omega_{c i}$. At the Earth's magnetopause, we have $T_{e} \sim 1 \mathrm{MK}$ and $B \sim 120 \mathrm{nT}$, so $c_{s} \approx 90 \mathrm{~km} / \mathrm{s}$, $\Omega_{c i} \approx 12 \mathrm{rad} / \mathrm{s}$, and $\rho_{s} \approx 7.5 \mathrm{~km}$.

The observations displayed in Fig. 2 (second panel left) are discussed from right to left to better follow the penetration of the solitary structures into the magnetosphere. The figure shows that there is the excitation of electrostatic (ES) turbulence in the dc electric field with a dominant wavelength of $1.5-2 \mathrm{~km}$ or $(0.2-0.25) \rho_{s}$. This ES wave, which will be shown to correspond to a zonal flow, is observed at the edge of the magnetosheath plasma, where
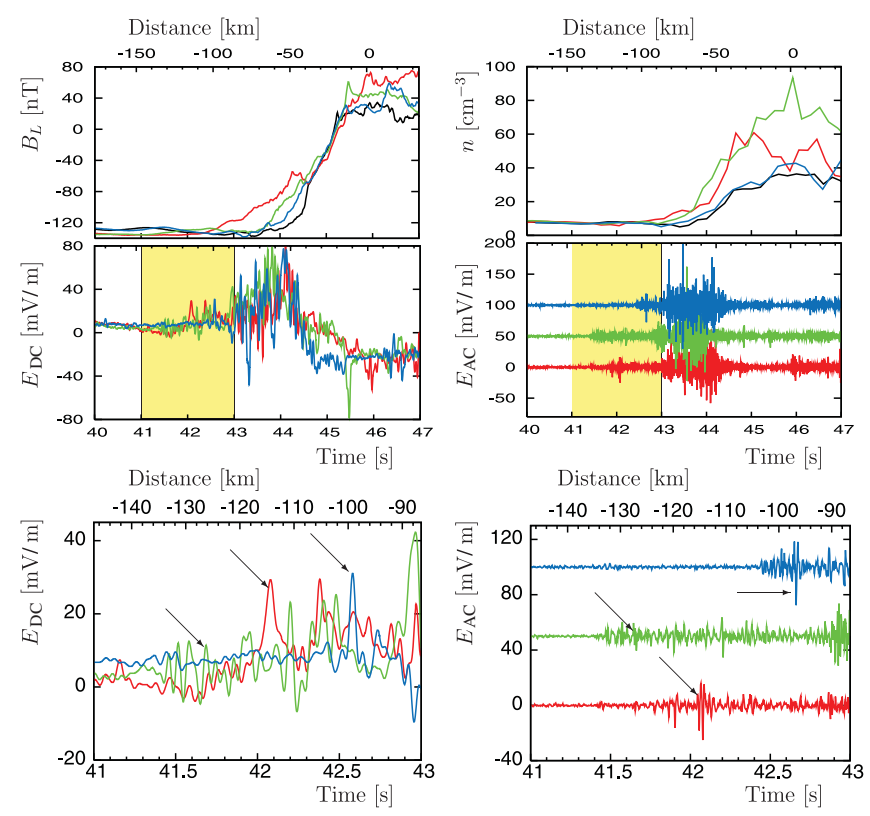

FIG. 2 (color). Cluster observations of a thin magnetopause, taken on 30 March, 2002, between 13:11:40 and 13:11:46 UT. Clockwise, the top four figures display the $L$ component (component of maximum variance) of the magnetic field in an LMN coordinate system (the $N$ component is normal to the boundary), the equilibrium plasma density as derived from the spacecraft potential, the ac electric field ( $N$ component, $20-180 \mathrm{~Hz}$ ), and the dc electric field ( $N$ component, $0-20 \mathrm{~Hz}$ ), all versus time (bottom scale) or distance (upper scale). Color coding is the same as in Fig. 1. In the graphs showing the ac electric field, the fields for $\mathrm{C} 3$ and $\mathrm{C} 4$ have been shifted by 50 and $100 \mathrm{mV} / \mathrm{m}$, respectively, for clarity. The $B_{L}$ graph shows that the peak current within the current layer occurs just upstream of the main density gradient (top panel right), while the electric field graphs exhibit, respectively, that the dc electric field perturbation and the main burst of ac power lie on the inner edge of the current layer and in the density gradient. The highlighted regions in the overall electric field graphs have been enlarged in the bottom two frames. In both the enlarged field graphs, a solitary wave packet, indicated by arrows, can be seen to separate itself from the bulk of the turbulence and move down the plasma density gradient into a previously turbulence-free region. Its direction follows from the order of the spacecraft boundary crossings ( $\mathrm{C} 4, \mathrm{C} 1, \mathrm{C} 2, \mathrm{C} 3)$.

the plasma electron density $n$ drops from about 60 (magnetosheath) to $6 \mathrm{~cm}^{-3}$ (magnetopause) over about $75 \mathrm{~km}$, or $10 \rho_{s}$. This corresponds to a relative density gradient $\left(\rho_{s} / n\right) \nabla n$ of up to 0.9 . The slow ES wave mainly occurs in regions where $(1 / n) \nabla n$ is not too large; it does not grow in regions where $n$ is small [and thus $(1 / n) \nabla n$ is large]. In the magnification of the highlighted region (bottom panel left), a solitary wave structure breaking away from the main ES wave is indicated by arrows. From the known separation of the spacecraft and the order in which they crossed the magnetopause, we infer that this structure, with an accompanying drift wave packet, moves down the gradient into the magnetosphere at a speed of roughly $8-9 \mathrm{~km} / \mathrm{s}$, or 
$(0.08-0.1) c_{s}$. Its size is 6 to $7 \mathrm{~km}$, or $(0.8-0.9) \rho_{s}$, and increases during propagation.

The slow ES wave is accompanied by strong drift mode turbulence in the ac electric field (second and third panel right), also associated with the ion density gradient within the magnetopause boundary layer. A peak electric field of $20-30 \mathrm{mV} / \mathrm{m}$ and an average wavelength of $500 \mathrm{~m}$ combine to give a peak potential of roughly $2 \mathrm{~V}$ for the ac turbulence $\left(e \tilde{\phi} /\left(k_{B} T_{e}\right) \sim 0.025\right.$ for $T_{e} \sim 1 \mathrm{MK}$, where $\tilde{\phi}$ denotes the ac potential and $k_{B}$ is Boltzmann's constant). Downstream from the magnetopause, a lower amplitude ac signal can be seen penetrating the magnetosphere to different depths for each spacecraft. Moving wave packets can be observed in this signal; their position and speed coincide with those of the solitary structures in the dc field. These wave packets maintain their coherence during the passage of all four Cluster spacecraft, while different wave packets are seen to move independently of each other. The observed breakup of the ac turbulence into wave packets follows from the nature of the wave-collapse instability [12] of electrostatic drift modes. The dimensions of these wave packets are set by the zonal flow characteristic wave length, which is of the order of $\rho_{s}$, in agreement with the observed structure size.

Numerical simulations have been carried out to investigate the mechanisms governing the emergence and propagation of the aforementioned solitary structures. We have used the so-called wave-kinetic approach [16-19], as it is particularly suited to describe broadband turbulence in the spectrum of the high-frequency wave components. It also allows one to follow the propagation of individual spectral modes, thus providing deeper insight into the wave evolution.

The numerical code used for our simulations has been based on the wave-kinetic approach to drift wave-zonal flow interactions in magnetized plasmas $[6,7,20,21]$, as found in, e.g., tokamaks. This approach is centered around the wave mode density $N(t, x, k)$, of which the evolution is given by a Boltzmann-like equation. In the numerical implementation, this distribution is approximated by a collection of macro-particles representing individual wave modes. A detailed description of the numerical model can be found elsewhere [7]. In our simulations, we have used a broadband distribution of drift waves in a plasma with a density profile modeled after the density profile observed by the Cluster satellites at the magnetopause boundary. The initial drift mode distribution is homogeneous in $(x, y)$-space, and Gaussian in $\left(k_{x}, k_{y}\right)$-space $(\mathbf{k}=$ $\left(k_{x}, k_{y}\right)$ denotes the drift mode wave vector), with a mean $k$ value of $3 / \rho_{s}$ and a spread of $1 / \rho_{s}$. Simulation results are displayed in Fig. 3: the electrostatic field $E$, representing the zonal flow, and (the distribution of) the longitudinal wave numbers $k_{x}$ of the drift modes, representing the fast drift wave turbulence, are plotted versus the longitudinal coordinate $x$. The stacked plots are therefore the numerical counterpart of the sampling of the magnetospheremagnetopause region at different times by the individual
Cluster spacecraft, and correspond directly to the bottom panels of Fig. 2.

In the simulation results, we first observe the excitation of a dc electrostatic wave, the zonal flow, at the plasma edge through the wave-collapse instability of the drift modes. This zonal flow has a wavelength of about $(0.5-$ 0.7) $\rho_{s}$, which is within a factor of 2 of the observed value of about $0.25 \rho_{s}$. Zonal flows can only grow when the imaginary part of their frequency, denoted by $\gamma$, is nonzero and in regions where there is good resonance between fluctuations in the drift mode density and the zonal flow. Using Ref. [6], the first condition translates to $3 k_{x}^{2}<1+$ $k_{y}^{2}$, while the second (resonance) condition requires the diamagnetic drift speed $V_{d}=\left(-c_{s} \rho_{s} / n\right) \nabla n$ to satisfy $\left|V_{d}\right|<$ $|\gamma / q|=\left|e \tilde{\Phi} /\left(k_{B} T_{e}\right)\right|\left(1+k_{x}^{2}+k_{y}^{2}\right)^{3 / 2}\left(1-3 k_{x}^{2}+k_{y}^{2}\right)^{1 / 2} /$ $\left(2\left|k_{x}\right|\right)$, where $q$ denotes the zonal flow wave number. This explains why initially no zonal flow develops in regions where $n$ is small, both in observations and simulations, because $V_{d}$ is too large there and there is no resonant coupling between the drift modes and the zonal flow.
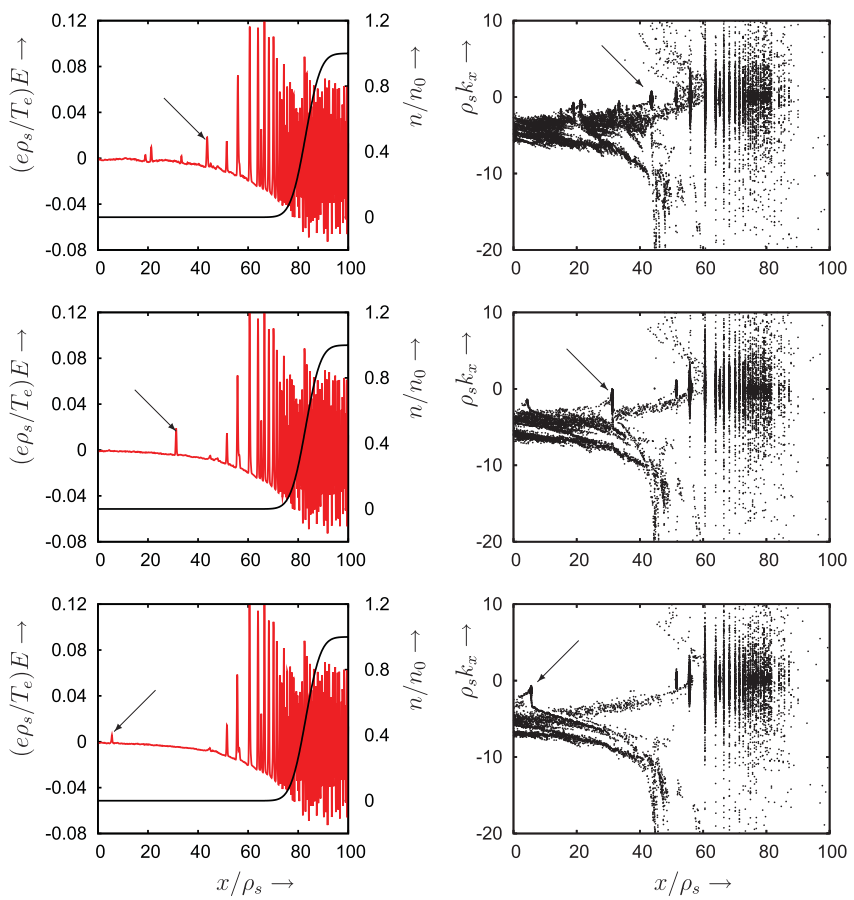

FIG. 3 (color). Simulation results for a thin magnetopause, where the background plasma density profile has been chosen to match the sudden rise in the plasma density at the magnetopause boundary. Snapshots of the slow electrostatic field (representing the zonal flow) and the drift mode phase space (representing the fast drift wave turbulence) were taken at $\Omega_{c i} t=500$ (top), 666 (middle), and 833 (bottom). The left column shows the slow electrostatic field (red) and the background plasma density (black), while on the right the drift mode phase space can be seen. The excitation of a zonal flow through the modulational instability only occurs for larger $x$, where the relative density gradient is shallow. A solitary wave structure breaking off the main zonal flow and drifting down the density gradient is indicated by arrows. 
This explains both why the zonal flow growth is largest for smallest inverse scale length $1 / L_{n}=V_{d} /\left(c_{s} \rho_{s}\right)$, and why the zonal flow tends to propagate towards steeper density gradients. For a fixed drift wave amplitude, large density gradients suppress the instability.

The simulations show solitary structures breaking away from the main zonal flow region and propagating independently into regions where no zonal flow developed earlier, retaining their identity for quite a long time. The size of these structures is about $(0.7-1.0) \rho_{s}$, which is close to the observed size of $(0.8-0.9) \rho_{s}$. As in the observations, the structures are stretched during propagation. Their speed increases from $0.02 c_{s}$ to $0.05 c_{s}$, which is within a factor 2 from the observations, as they move further down the density gradient. In the $\left(x, k_{x}\right)$-space for the drift modes, there is clumping of drift modes as a result of the wave-collapse instability. Quasiparticles from the same clump stay together for a long time, while the clumps may propagate independently from each other. These effects are all directly seen in the observations as well.

We can use the above resonance condition to estimate a threshold value for the instability in the observations. From the spacecraft data, we note that there is no instability for $\rho_{s}(\nabla n) / n \geq 0.2 \pm 0.1$. Inserting this into the resonance condition, this requires the drift mode amplitude to satisfy $e \tilde{\Phi} /\left(k_{B} T_{e}\right) \approx 0.02 \pm 0.01$. The observed amplitude of $e \tilde{\Phi} /\left(k_{B} T_{e}\right) \approx 0.025(\sim 2$ Volts $)$ falls well within this interval.

As explained in Ref. [7], the formation of these solitary structures can be explained from the interplay between the $\mathbf{E} \times \mathbf{B}$ drift $V_{0} \propto \partial \bar{\phi} / \partial x$ (where $\bar{\phi}$ is the zonal flow potential) and the diamagnetic drift $V_{d} \propto-\left(1 / n_{0}\right) \partial n_{0} / \partial x$, where $n_{0}(x)$ denotes the equilibrium plasma density. The $\mathbf{E} \times \mathbf{B}$ drift follows the perturbation in $\bar{\phi}$ and causes the solitary structure to stay together, trapped in a period of the zonal flow, while the back action that the drift waves exert on the background plasma tends to enhance the zonal flow and thus improve confinement of the drift waves. On the other hand, the diamagnetic drift acts in the same direction for all drift modes with the same sign of $k_{y}$, and increases with the relative density gradient. This drift causes drift modes on a density slope to move apart, thus opposing the effect of the $\mathbf{E} \times \mathbf{B}$ drift. Initially, the diamagnetic drift only causes clumps of drift modes to move away from each other and towards regions with even steeper density gradients, explaining the observed behavior of the solitary structures. However, when the relative density gradient becomes sufficiently large, the structures themselves are ripped apart. All these aspects of the drift wave-zonal flow interactions are seen in both observations and simulations. This good correspondence proves that the dc electrostatic waves in the observations are indeed zonal flows, and explains the mechanisms behind the solitary structures seen in the satellite observations.
In conclusion, we have studied Cluster observations displaying the spontaneous emergence of coherent solitary structures from broadband ac turbulence at the magnetopause boundary layer. The unique capabilities of the four Cluster spacecraft have allowed the identification of the solitary structures as separate entities. The study of their evolution, as they propagate down the plasma density gradient associated with the magnetopause and penetrate the magnetosphere, has been achieved as a result of the sequential sampling by each spacecraft. Numerical simulations have provided the interpretation of these structures in terms of a nonlinear phenomenon known as wave collapse. The drift mode turbulence, also observed by Cluster, is the driving force behind this phenomenon.

The authors would like to thank the STFC Centre for Fundamental Physics for its support.

*Also at University of Strathclyde, Glasgow, United Kingdom.

[1] J.S. Russell, Report of the Fourteenth Meeting of the British Association for the Advancement of Science, York, 1844 (BAAS, London, 1845), p. 311, Plates XLVII-LVII.

[2] Editorial: Soliton Wave Receives Crowd of Admirers, Nature (London) 376, 373 (1995).

[3] A. Hasegawa and K. Mima, Phys. Fluids 21, 87 (1978).

[4] A. Hasegawa and M. Wakatani, Phys. Rev. Lett. 50, 682 (1983).

[5] W. Horton and A. Hasegawa, Chaos 4, 227 (1994).

[6] A.I. Smolyakov, P. H. Diamond, and V.I. Shevchenko, Phys. Plasmas 7, 1349 (2000).

[7] R. Trines et al., Phys. Rev. Lett. 94, 165002 (2005).

[8] V. I. Petviashvili and O. A. Pokhotelov, Solitary Waves in Plasmas and in the Atmosphere (Gordon and Breach, Philadelphia, 1992).

[9] C.P. Escoubet, M. Fehringer, and M. Goldstein, Ann. Geophys. 19, 1197 (2001), http://www.ann-geophys.net/ 19/1197/2001/.

[10] J. De Keyser et al., Space Sci. Rev. 118, 231 (2005).

[11] I. Silin, J. Büchner, and A. Vaivads, Phys. Plasmas 12, 062902 (2005).

[12] P. A. Robinson, Rev. Mod. Phys. 69, 507 (1997).

[13] G. Gustafsson et al., Space Sci. Rev. 79, 137 (1997).

[14] M. Dunlop et al., Ann. Geophys. 19, 1449 (2001), http:// www.ann-geophys.net/19/1449/2001/.

[15] A. Pedersen et al., Ann. Geophys. 19, 1483 (2001), http:// www.ann-geophys.net/19/1483/2001/.

[16] R. Bingham et al., Phys. Lett. A 220, 107 (1996).

[17] R. Bingham et al., Phys. Rev. Lett. 78, 247 (1997).

[18] L. O. Silva et al., IEEE Trans. Plasma Sci. 28, 1202 (2000).

[19] J. T. Mendonça, R. Bingham, and P. K. Shukla, Phys. Rev. E 68, 016406 (2003).

[20] N. Mattor and P. H. Diamond, Phys. Plasmas 1, 4002 (1994).

[21] A.I. Smolyakov and P.H. Diamond, Phys. Plasmas 6, 4410 (1999). 